\title{
ARTICLE
}

Received 4 Dec 2014 | Accepted 26 Mar 2015 | Published 11 May $2015 \quad$ DOl: 10.1038/ncomms8047

\section{Theory of Floquet band formation and local pseudospin textures in pump-probe photoemission of graphene}

\author{
M.A. Sentef ${ }^{1,2}$, M. Claassen ${ }^{3}$, A.F. Kemper ${ }^{4}$, B. Moritz ${ }^{1,5}$, T. Oka $^{6}$, J.K. Freericks ${ }^{7}$ \& T.P. Devereaux ${ }^{1,3}$
}

Ultrafast materials science promises optical control of physical properties of solids. Continuous-wave circularly polarized laser driving was predicted to induce a light-matter coupled state with an energy gap and a quantum Hall effect, coined Floquet topological insulator. Whereas the envisioned Floquet topological insulator requires high-frequency pumping to obtain well-separated Floquet bands, a follow-up question regards the creation of Floquet-like states in graphene with realistic low-frequency laser pulses. Here we predict that short optical pulses attainable in experiments can lead to local spectral gaps and novel pseudospin textures in graphene. Pump-probe photoemission spectroscopy can track these states by measuring sizeable energy gaps and Floquet band formation on femtosecond time scales. Analysing band crossings and pseudospin textures near the Dirac points, we identify new states with optically induced nontrivial changes of sublattice mixing that leads to Berry curvature corrections of electrical transport and magnetization.

\footnotetext{
${ }^{1}$ Stanford Institute for Materials and Energy Sciences (SIMES), Stanford University and SLAC National Accelerator Laboratory, Menlo Park, California 94025, USA. ${ }^{2}$ HISKP University of Bonn, Bonn 53115, Germany. ${ }^{3}$ Geballe Laboratory for Advanced Materials, Stanford University, Stanford, California 94305 , USA. ${ }^{4}$ Lawrence Berkeley National Lab, 1 Cyclotron Road, Berkeley, California 94720, USA. ${ }^{5}$ Department of Physics and Astrophysics, University of North Dakota, Grand Forks, North Dakota 58202, USA. ${ }^{6}$ Department of Applied Physics, University of Tokyo, Tokyo, 113-8656, Japan. ${ }^{7}$ Department of Physics, Georgetown University, Washington, DC 20057, USA. Correspondence and requests for materials should be addressed to M.A.S. (email: michael.sentef@hiskp.uni-bonn.de) or to T.P.D. (email: tpd@stanford.edu).
} 
T he ultrafast optical manipulation of materials by femtosecond laser pulses is rapidly becoming a major guiding theme in condensed matter physics ${ }^{1-3}$. At the same time, the quest for novel topological states of matter triggered enormous research activity since the discovery of topological insulators ${ }^{4}$. Merging both of these vibrant fields, a recent work reported the coupling of short laser pulses to surface Dirac fermions in the topological insulator $\mathrm{Bi}_{2} \mathrm{Se}_{3}$ (ref. 5). This work demonstrated the creation of Floquet-like sidebands during irradiation as well as the opening of a small band gap at the surface state Dirac point for circular light polarization.

Time-reversal symmetry protects massless Dirac fermions on the surface of topological insulators ${ }^{6,7}$ and, in combination with inversion symmetry, also in graphene in the absence of spin-orbit coupling ${ }^{8}$. In a milestone paper, Haldane envisioned that breaking either or both of these symmetries would open a gap at the Dirac points in graphene, allowing one to tune between a trivial insulator and a Chern insulator ${ }^{9}$. While equilibrium band gap engineering has become a major theme since the first synthesis of monolayer graphene, it was only recently proposed that circularly polarized, high-frequency laser light could turn trivial equilibrium bands into topological nonequilibrium Floquet bands $^{10-25}$, coined Floquet topological insulator (FTI).

The FTI concept is based on two things: first, in the limit of continuous laser driving at frequency $\Omega$, the temporal periodicity allows one to employ a repeated quasi-energy zone scheme with a temporal Brillouin zone of size $\Omega$. Second, in the high-frequency limit, defined by $\Omega$ being larger than the electronic bandwidth, these repeated zones contain well-separated copies of the original electronic bands spaced by integer multiples $n \Omega$, the so-called Floquet sidebands. The effect of the laser on the original $n=0$ band manifold is perturbative in $1 / \Omega$. If the laser is circularly polarized, time-reversal symmetry is broken and an energy gap opens at the Dirac points in graphene due to the fact that photon emission and absorption processes do not commute. The FTI concept then follows from an exact mapping of driven graphene to the Haldane model, leading to a well-defined nonzero Chern number.

Whereas this envisioned high-frequency strong pumping limit that is required for nontrivial topological states is currently experimentally unattainable, a natural follow-up question regards the engineering of local spectral gaps in realistic pump-probe experiments. This leads to the question on which time scales the quasi-steady Floquet regime can be reached when continuouswave driving is replaced by a short laser pulse. Moreover, it requires the investigation of the low-frequency regime, in which
$1 / \Omega$ perturbation theory is not applicable. In this regime, the overlap of different Floquet sidebands prevents a global topological classification of states.

In this work, we address these problems by simulating the realtime development of single-particle energy gaps in graphene coupled to short laser pulses, using realistic parameters for timeresolved, angle-resolved photoemission spectroscopy (tr-ARPES). We show that the tr-ARPES band structure shows well-defined Floquet bands provided that a hierarchy of time scales is fulfilled between the duration of the pump-pulse, the duration of the probe-pulse, and the laser period: $\sigma_{\text {pump }}>\sigma_{\text {probe }} \gg 2 \pi \hbar / \Omega$. We predict the opening of a Dirac point gap and the formation of Floquet sidebands that form on femtosecond time scales. An important difference to the high-frequency limit arises from the overlap of Floquet sidebands. At frequency-dependent critical driving field strengths, we find a sequence of level crossings and energy gap closings at the Dirac points. The analysis of snapshots of pseudospin textures near the Dirac points allows us to identify optically induced nontrivial changes of sublattice mixing at these level crossing points, that manifest themselves in Berry curvature corrections of electrical transport and magnetization. Even though a global topology cannot be assigned to the low-frequency driven states, we show that the analysis of level crossings and energy gap closings leads to a classification scheme in terms of local gaps and Berry curvatures.

\section{Results}

Haldane model. To set the stage for our results, we briefly outline the basic ingredients for the low-energy physics of Haldane's equilibrium model. We start from two Dirac cones with effective Hamiltonian $v_{\mathrm{D}}\left(q_{x} \sigma_{x} \otimes \tau_{z}+q_{y} \sigma_{y} \otimes I\right)$. Here $v_{\mathrm{D}}$ is the Dirac point velocity, the Pauli matrices $\boldsymbol{\sigma}$ label pseudospin arising from the graphene sublattices $\mathcal{A}$ and $\mathcal{B}, \tau$ labels the valley degree of freedom corresponding to the Dirac cones around $K$ and $K^{\prime}$, and $\boldsymbol{I}$ is the $2 \times 2$ identity matrix. Electron spin can be neglected in the absence of spin-orbit coupling. Momentum $\mathbf{q}$ is measured from the respective Dirac points. The pseudospin content $\mathbf{P}(\mathbf{q})$ essentially measures orbital band content (see Supplementary Note 1). For instance, a pseudospin pointing along the $+z$ (up) direction means that the band is predominantly of $\mathcal{A}$ sublattice character, while a pseudospin pointing along the $-z$ (down) direction indicates mainly $\mathcal{B}$ sublattice character. Together with the winding of the $P_{x}$ and $P_{y}$ in-plane pseudospin components around the Dirac points, $P_{z}$ determines the local Berry curvature $\mathcal{F}(\mathbf{q})=\left(\partial_{q_{x}} \mathbf{P}(\mathbf{q}) \times \partial_{q_{y}} \mathbf{P}(\mathbf{q})\right) \cdot \mathbf{P}(\mathbf{q})^{26}$.
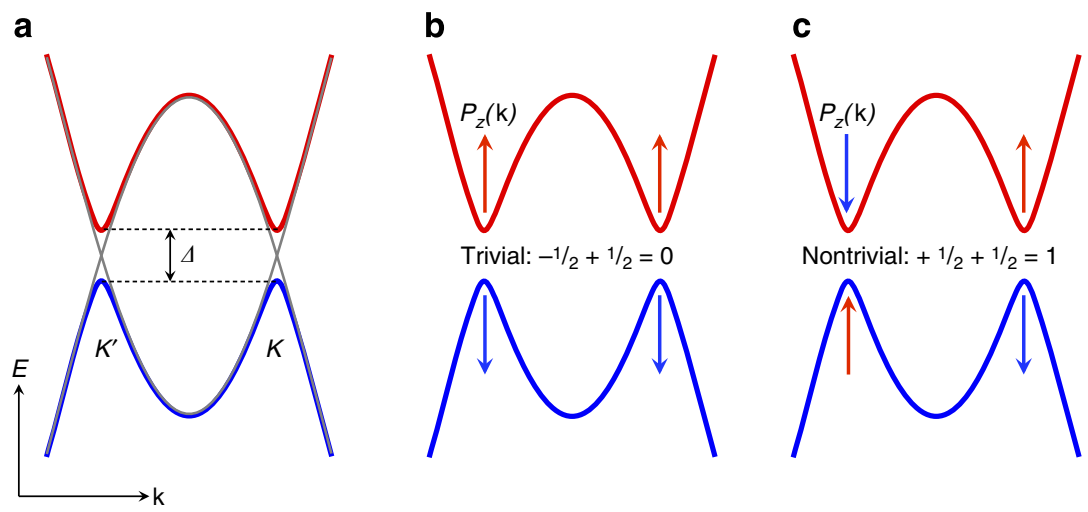

Figure 1 | Graphene with band gap. (a) Graphene band energy ( $E$ ) versus momentum (k) dispersion with a band gap $\Delta$ at the Dirac points. (b) Trivial gap structure in Haldane's model: The pseudospin $P_{z}(\mathbf{k})$, indicated by up and down arrows, points in the same direction at both Dirac points $K$ and $K^{\prime}$. The winding number contributions cancel. This gap structure appears if inversion symmetry is broken, and time-reversal symmetry is intact. (c) Nontrivial gap structure: $P_{z}(\mathbf{k})$ points in opposite directions at $K$ and $K^{\prime}$, leading to a nonzero Chern number +1 or -1 . This gap structure appears if time-reversal symmetry is broken and inversion symmetry is intact. 

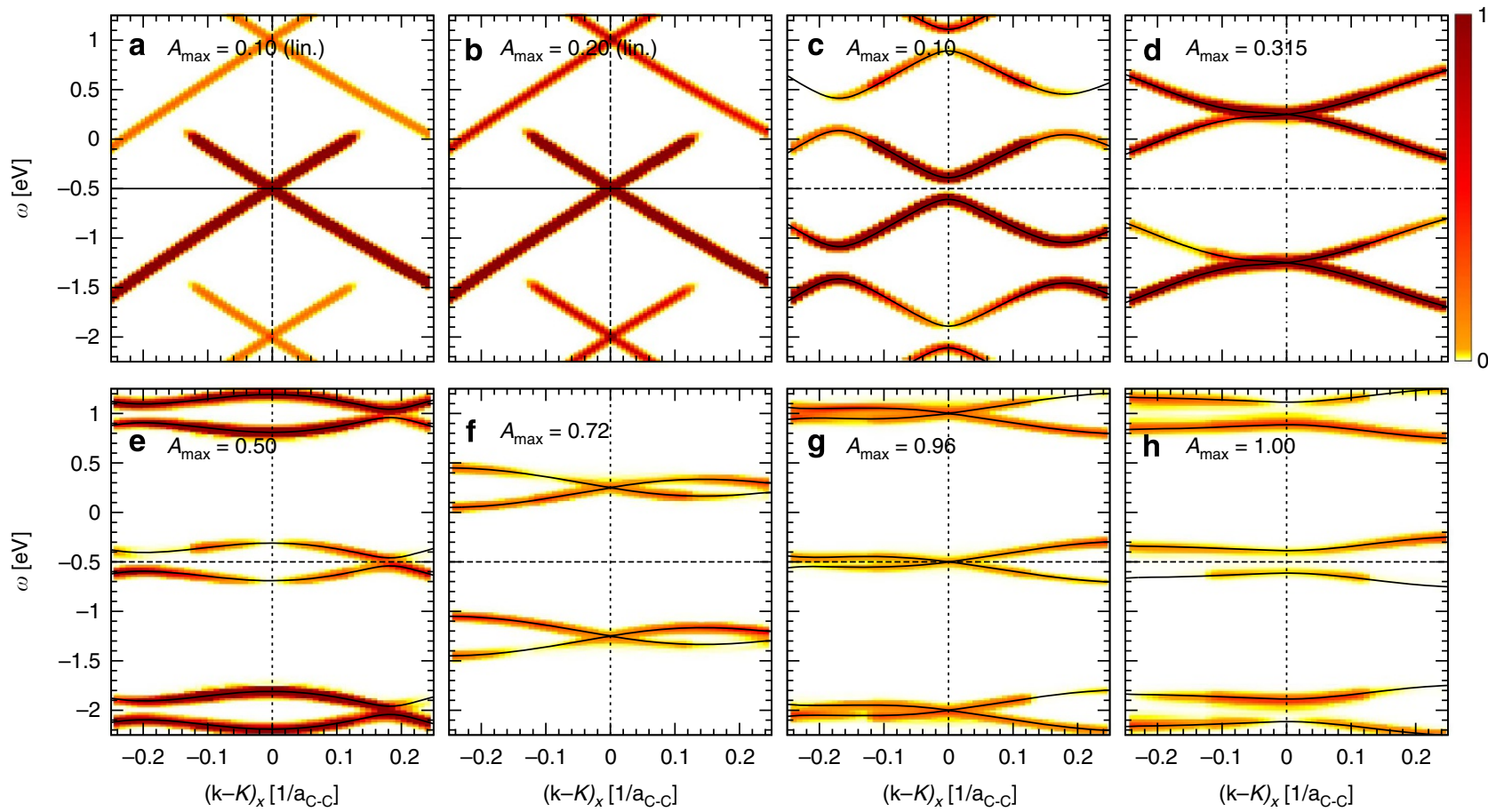

Figure 2 | Transient angle-resolved photoemission spectrum. (a-h) tr-ARPES spectral intensity of irradiated graphene at peak field (delay time $\Delta t=0$ ) versus binding energy $\omega$ and momentum $(k-K)_{x}$ near the Dirac $K$ point (vertical dashed lines) with Dirac point energy at $-0.5 \mathrm{eV}$ (horizontal dashed lines) on a momentum cut along the $\Gamma-K-K^{\prime}$ direction. The colour scheme varies between 0 (white) and maximal (red) spectral intensity. The pump laser field is linearly polarized in panels (a) and (b), and circularly polarized in panels (c-h). The pump-pulse field strength $A_{\text {max }}$ is varied between panels, with $A_{\max }$ values and laser polarizations as indicated. The driving frequency is $1.5 \mathrm{eV}$ for all panels. The solid curves show the corresponding quasi-static Floquet band structures. The linearly polarized laser in panels (a) and (b) does not induce a band gap, whereas the circularly polarized laser in panels (c-h) first induces a band gap at the Dirac point and then leads to a sequence of level crossings (panels (d) and (f)) and gap closing (g) as the field strength is increased.

In Haldane's model, an effective mass term $m \sigma_{z} \otimes \tau_{\kappa}$ leads to an energy gap $\Delta=2 m$ at the Dirac points (Fig. 1a). Its relative sign between $K$ and $K^{\prime}$ is determined by $\tau_{\kappa}$ and depends on its origin: If the gap is induced by introducing a staggered sublattice potential breaking inversion symmetry, $\tau_{\kappa}=\tau_{0}$, implying that the effective mass term has the same sign at $K$ and $K^{\prime}$, and the out-ofplane pseudospin component $P_{z}$ is the same at both Dirac points (Fig. 1b). By contrast, if the gap originates from breaking timereversal symmetry, $\tau_{\kappa}=\tau_{z}$, hence $P_{z}$ points in opposite directions (Fig. 1c).

Time-resolved photoemission spectra of driven graphene. We now come to the discussion of our nonequilibrium results. We start from the minimal honeycomb-lattice tight-binding model of graphene. We drive this system by coupling to a time-dependent, spatially homogeneous electric field modelled as a timedependent vector potential $\mathbf{A}(t)$, which couples to the electrons via Peierls substitution. The relativistic magnetic component of the light field is neglected. The pump-pulse has a temporal width $\sigma_{\text {pump }}=165 \mathrm{fs}$, photon frequency $\Omega=1.5 \mathrm{eV}$ (laser period of $2.8 \mathrm{fs}$ ), with linear or circular light polarization, corresponding to a femtosecond pump-pulse. The field strength is given by $A_{\max }$, which is measured in units of the inverse carbon-carbon distance. For graphene, the conversion to the peak electric field strength is $E_{\max }=A_{\max } \times 1,060 \mathrm{mV} \AA^{-1}$ for $\Omega=1.5 \mathrm{eV}$. We track the time- and momentum-resolved single-particle spectrum of the pump-driven electrons using a short 26 fs probe-pulse that emits photoelectrons and thereby generates a photocurrent ${ }^{27,28}$, as measured experimentally with tr-ARPES (see Methods).
We first characterize the nonequilibrium band structures using tr-ARPES spectra. Fig. 2 shows the tr-ARPES spectra on a momentum cut along the $\Gamma-K-K^{\prime}$ direction near $K$ at peak field ( $\Delta t=0 \mathrm{fs}$ ). We first perform a calculation using pump pulses with linear polarization along the $k_{x}$ direction for two different field strengths (Fig. 2a,b). One can see the formation of Floquet sidebands, but since the pump preserves time-reversal symmetry, the spectrum remains gapless at the Dirac point energy. The main effect of the linearly polarized pump is a shift of the Dirac point location from $K$ towards $K^{\prime}$, which increases with increasing field strength. This Dirac point shift is due to the nonlinearity of bands and does not happen for perfect Dirac cones.

Floquet spectrum and level crossings. Next, we turn to circular light polarization, thereby breaking time-reversal symmetry. In Floquet theory, the quasi-static eigenvalue spectrum at finite driving field $A$ shows copies of the original bands shifted by integer multiples of $\Omega$, the so-called Floquet sidebands. Energy gaps of $n$-th order in the field open at avoided level crossings of sidebands which differ by $n$ photon energies. For circular light, an energy gap of second order in the field opens at the Dirac point. In our tr-ARPES simulation, for a moderate field strength and $1.5 \mathrm{eV}$ photons, an energy gap exceeding $100 \mathrm{meV}$ at $K$ is induced, accompanied by avoided level crossing gaps nearby (Fig. 2c). Due to the aforementioned hierarchy of time scales, we observe an excellent agreement of tr-ARPES spectra and the quasi-static Floquet band structure obtained by diagonalizing the Floquet Hamiltonian involving large numbers of sidebands (solid lines, see Supplementary Material). 
a

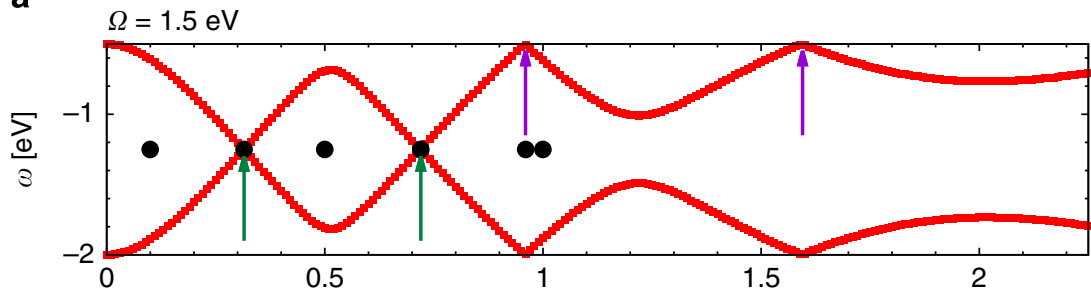

b
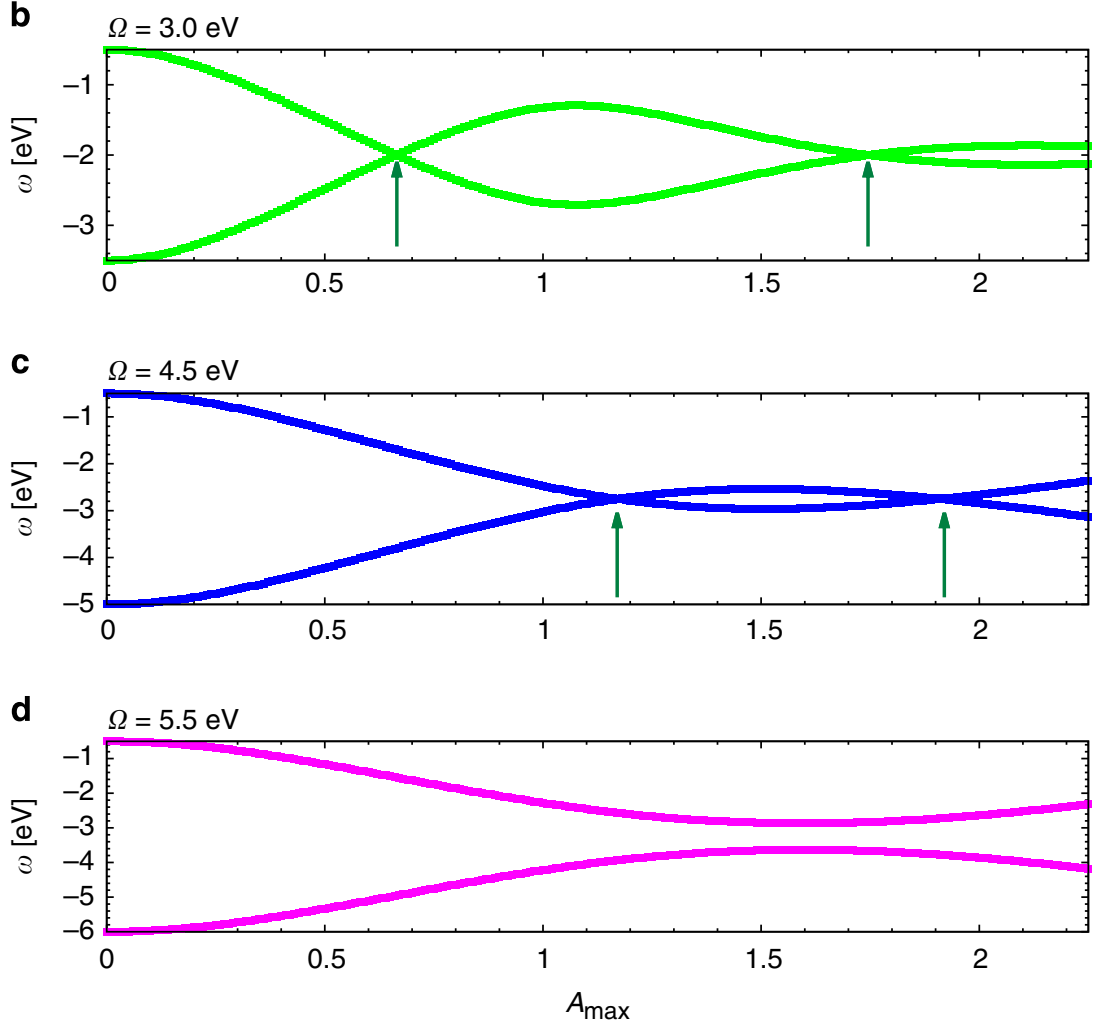

Figure 3 | Gap opening and level crossings in Dirac point Floquet spectra. First two negative Floquet quasi-energies $\omega$ at the Dirac points, shown below the Dirac point energy $\left(-0.5 \mathrm{eV}\right.$ ) as a function of field strength $A_{\max }$ for increasing driving frequencies varied between panels (a-d), as indicated. The level crossings between the two Floquet bands are indicated by green arrows, the gap closings at higher field strength for $\Omega=1.5 \mathrm{eV}$ are indicated by violet arrows. Black circles in panel (a) indicate the field strengths used in Fig. 2c-h. The sequence of level crossings and gap closings implies nontrivial changes in the pseudospin contents due to changes in the sublattice mixing of orbital band contents.

At larger field strength (Fig. 2d), the Floquet bands move closer to each other and cross. They separate again and the Dirac point energy gap decreases (Fig. 2e). At even larger fields, there is another crossing between Floquet bands (Fig. 2f) before the Dirac point gap closes (Fig. $2 \mathrm{~g}$ ) and eventually reopens (Fig. $2 \mathrm{~h}$ ). At the largest field strength shown here $\left(A_{\max }=1.00\right)$, the bands are almost flat, indicating that the ac Wannier-Stark limit is approached. This creation of flat Wannier-Stark bands in the strong driving limit impedes the continuous growth of the gap with increasing field strength.

To analyse the pump photon frequency dependence of the Dirac point level crossings and gap closing in more detail, we show in Fig. 3 the first two negative Floquet eigenvalues tracking the position of the first two Floquet bands below $E_{D}$. Fig. 3a shows the initial gap opening at $\Omega=1.5 \mathrm{eV}$, which is quadratic in the field at small $A_{\max }$, followed by two level crossings between the two Floquet bands indicated by two arrows. The gap at the Dirac point is then closed, indicated by the third arrow. For $\Omega=3.0 \mathrm{eV}$, the field range between the two Floquet band level crossings increases (Fig. 3b), then decreases at $\Omega=4.5 \mathrm{eV}$, and finally vanishes for $\Omega=5.5 \mathrm{eV}$. The initial quadratic gap opening is the same for all photon frequencies due to the linearity of the graphene bands near the Dirac points. The differences between different photon frequencies at larger fields then arise from the nonlinearity of the bands further away from the Dirac points, which is specific to graphene.

Local pseudospin textures near Dirac points. We now turn to the discussion of local pseudospin content. Fig. $4 \mathrm{a}-\mathrm{c}$ present false color plots of the momentum-resolved pseudospin contents near the Dirac points for the driven system at $\Omega=1.5 \mathrm{eV}$. At small field before the first level crossing (Fig. 4a), the $P_{x}$ and $P_{y}$ components have two sign changes along a path around $K$, as expected for weakly driven graphene. This nodal structure is directly related to the $q_{x} \sigma_{x}+q_{y} \sigma_{y}$ term in the effective low-energy Hamiltonian introduced above, which shows that the $P_{x}$ component transforms like $q_{x}$ and the $P_{y}$ component transforms like $q_{y}$. We coin this state $\mathrm{S}_{1}$, with one nodal line and therefore a single pseudospin winding in the vicinity of the Dirac points. Importantly, the $P_{z}$ component changes sign between $K$ and $K^{\prime}$, which is consistent with breaking time-reversal symmetry. 


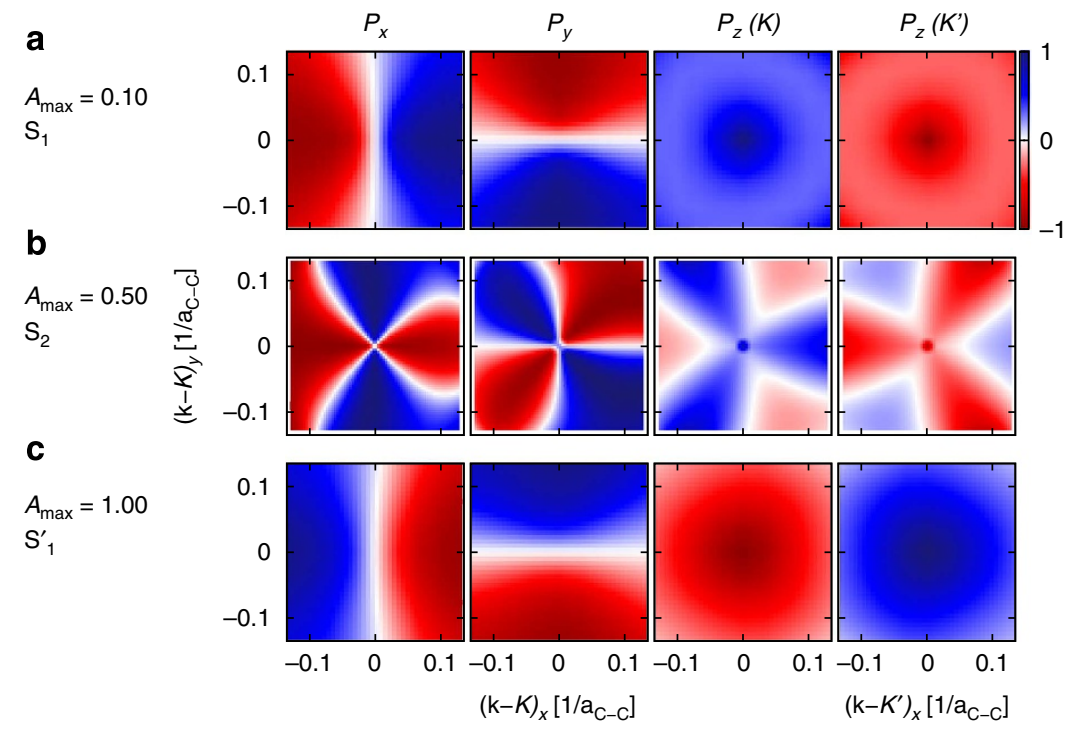

d

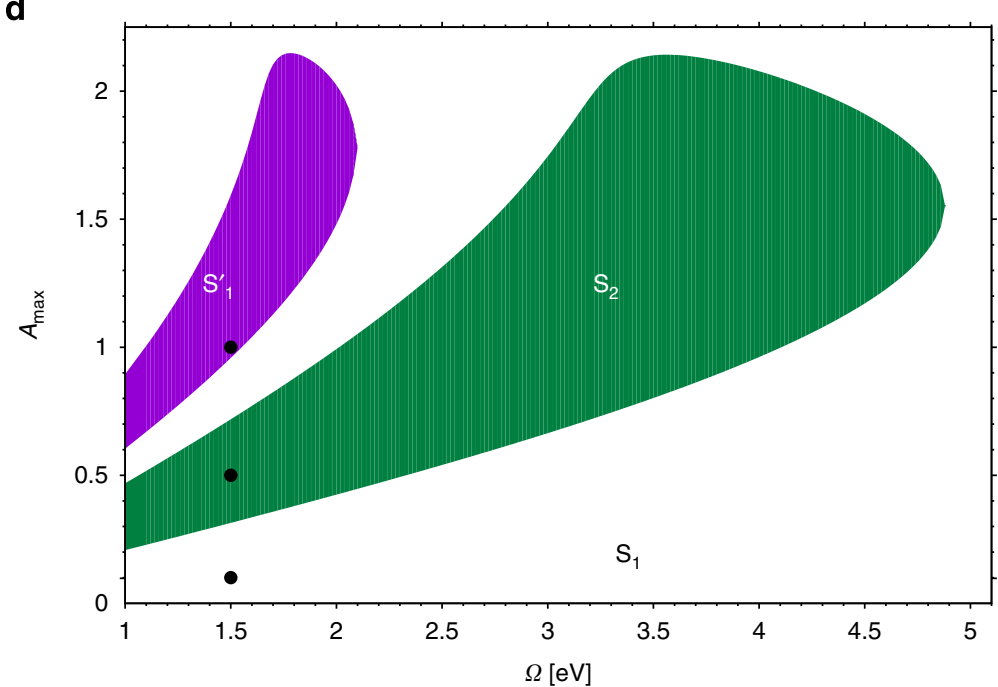

Figure 4 | Field-induced changes in local pseudospin textures. (a-c) Pseudospin textures of the components $P_{x, y, z}(\mathbf{k})$ near $K$ and $K^{\prime}$ at peak field (delay time $\Delta t=0$ ) for $\Omega=1.5 \mathrm{eV}$, with varying maximal field strengths $A_{\max }$ in panels (a-c) as indicated. The colour scheme varies between pseudospin component being -1 (red), $O$ (white) and +1 (blue). The pseudospin winding changes from $p$-wave in state $S_{1}$ (a) to fourfold nodal lines in $S_{2}$ (b) to $p$-wave in $S_{1}^{\prime}(\mathbf{c})$, with opposite pseudospin direction compared to the original $S_{1}$ state. The $P_{z}$ component changes sign between $K$ and $K^{\prime}$ since timereversal symmetry is broken. (d) Phase diagram of local pseudospin windings near the Dirac points as a function of driving field strength and frequency. The phase boundaries are obtained from the Dirac point level crossings and gap closings. Black circles indicate the parameter values used in panels (a-c).

When the field is increased through the first level crossing (Fig. $4 \mathrm{~b}$ ), the character of the local pseudospin textures changes. The effective mass term still changes sign between $K$ and $K^{\prime}$. Remarkably, the $P_{x}$ and $P_{y}$ components double their winding number, changing sign four times along a path around $K$. We call this state $S_{2}$, since it has two nodal lines in the vicinity of the Dirac point, which cross at the Dirac point. Although the corresponding ARPES spectrum for the same parameters (Fig. 2f) has only little spectral weight near $K$ in the Floquet bands close to $E_{D}$, the pseudospin texture is well-defined at all momenta considered here. Also, other sidebands have higher spectral weight, and each of the Floquet sidebands carries the same pseudospin information.

A similar texture is also obtained for Bernal stacked bilayer graphene $^{29}$ in a perpendicular electric field ${ }^{30}$. In this analogy, we stress that the doubled winding in bilayer graphene persists even when the energy gap induced by the electric field goes to zero. By contrast, in our case the doubled winding state $S_{2}$ vanishes when the Floquet sidebands cross, and gives way to a single winding state $S_{1}$ in the low-field limit. Also the effective perpendicular electric field generated by the circularly polarized light pulse in our work points in opposite directions at $K$ and $K^{\prime}$, in contrast to the static electric field applied in ref. 30, which corresponds to the Haldane mass term. In any case, the observation of state $S_{2}$ suggests the possibility to dynamically engineer effective models with higher pseudospin winding numbers, similarly to higherorder spin-orbital textures in topological insulators ${ }^{31}$.

When the field is increased further through the second level crossing and the gap closing, one obtains the pseudospin textures shown in Fig. 4c. Here all the pseudospin components are flipped compared to the ones in Fig. 4a, and we coin this flipped state with single pseudospin winding $S^{\prime}$.

Phase diagram at low-frequency driving. We are now in a position to discuss the phase diagram of local Berry curvatures, 
which follow from the pseudospin textures around the Dirac points, as a function of field strength and driving frequency. Fig. 4d shows the positions of the Floquet band level crossings indicating the transition to a pseudospin texture with a doubled number of nodal lines, as well as the Dirac energy gap closing leading to a state with inverted $P_{z}$ component. There is an upper frequency limit for the former state in the range of field strengths shown here. This is consistent with the fact that in the infinitefrequency limit, only states with $p$-wave pseudospin textures corresponding to $S_{1}$ and $S_{1}$ were found, which can be understood from the exact mapping to the static Haldane model in this limit ${ }^{32}$.

On one hand, the characterization of nonequilibrium states in terms of local pseudospin textures is restricted to momenta near the Dirac points by Floquet sideband level crossings. Such level crossings generically appear at low driving frequency $\Omega$ because different Floquet sidebands overlap if $\Omega$ is smaller than the electronic bandwidth. On the other hand, sideband level crossings at the Dirac point are the root cause of the appearance of the exotic pseudospin textures in Fig. 4b. The low-frequency behaviour of driven graphene is therefore more complicated, but also contains new states that are absent in the high-frequency limit. The complete evolution of the tr-ARPES spectra and pseudospin textures across the boundaries of the $S_{2}$ state is shown in the Supplementary Figs 1,2 and 3 for driving frequencies of $1.5,3.0$ and $4.5 \mathrm{eV}$, respectively, and discussed in Supplementary Note 2.

\section{Discussion}

Our combined results show that band gaps induced by breaking time-reversal symmetry in graphene are within reach under realistic experimental conditions. In particular, the achievable energy resolution for probe-photon energies which are sufficiently high to reach the Dirac points ${ }^{33,34}$ should allow for the detection of photoemission gap sizes exceeding $100 \mathrm{meV}$. The change in pseudospin texture near critical driving offers the exciting opportunity of optical manipulation of local Berry curvatures near Dirac points on ultrafast time scales. Moreover, the combination of broken inversion symmetry and broken timereversal symmetry opens up the possibility of controlling the valley degree of freedom and inducing different energy gaps at the two Dirac points ${ }^{35-38}$.

The spectroscopic detection of pseudospin textures requires access to orbital band content. To this end, hexagonal structures with inequivalent orbitals on the $\mathcal{A}$ and $\mathcal{B}$ sublattices having different photoemission probe-energy cross-sections could be examined. A candidate material for this purpose is hexagonal boron nitride. The demonstration of pseudospin imbalance at the two Dirac points by circularly polarized light in boron nitride would be intriguing. Alternatively, artificial hexagonal lattices with sublattice potentials have already been demonstrated with cold atoms ${ }^{39}$. Thus the proposed pseudospin textures could in principle also be realized in driven ultracold quantum gases ${ }^{40,41}$.

\footnotetext{
Methods

Methods summary. The simulations presented here start from a minimal tightbinding model of spinless electrons with nearest-neighbour hopping on the honeycomb lattice ${ }^{8,42}$. The pump-pulse drives the electrons via minimal coupling to a gauge field $\mathbf{A}(t)=A_{\max } p_{\sigma_{\text {pump }}}(t)\left(\sin (\Omega t) \mathbf{e}_{x}+\cos (\Omega t) \mathbf{e}_{y}\right)$, with a Gaussian shape function $p_{\sigma_{\text {pump }}}(t)=\exp \left(-\left(t-t_{\mathrm{p}}\right)^{2} /\left(2 \sigma_{\text {pump }}^{2}\right)\right)$ for a pulse of width $\sigma_{\text {pump }}$ centred around time $t_{\mathrm{p}}$, and photon frequency $\Omega$. The phase shift of $\pi / 2$ between the $x$ and $y$ components, represented by the unit vectors $\boldsymbol{e}_{x}$ and $\boldsymbol{e}_{y}$, describes circular light polarization. For comparison we also study linearly polarized light with vanishing $y$ component. Throughout this work we use units where $e=\hbar=c=1$. The electric field is $\mathbf{E}(t)=-\partial \mathbf{A}(t) / \partial t$, and we neglect the relativistic magnetic field of the laser pulse. This means that the electronic spin degree of freedom maintains its full degeneracy, and it is therefore not explicitly included in
}

our calculations. In particular, both the photoemission spectra and the pseudospin textures are identical for both physical spin species.

The tr-ARPES is computed from the trace of the nonequilibrium lesser Green function by a postprocessing step involving the probe-laser-pulse shape $s_{W}(t)$ with time resolution $W$, which leads to an effective tr-ARPES energy resolution $\propto 1 / \sigma_{\text {probe }}{ }^{27,28}$. The delay time of a pump peaked at $t_{\mathrm{p}}$ and probe peaked at $t_{\mathrm{pr}}$ is given by $\Delta t \equiv t_{\mathrm{pr}}-t_{\mathrm{p}}$.

Simulations are performed for an initial equilibrium sample temperature $T=116 \mathrm{~K}$. We typically use 500,000 $-1,500,000$ time steps for the computation of the time evolution operator, depending on the field parameters. This corresponds to a maximal time-step size of $0.0016 \mathrm{fs}$. Green functions for tr-ARPES measurements are sampled on a grid with 5,000-15,000 real-time steps and a maximal step size of $0.16 \mathrm{fs}$. The Dirac point velocity is given by $v_{\mathrm{D}}=4.2 \mathrm{eV} \mathrm{a} \mathrm{C}-\mathrm{C}$, where $\mathrm{a}_{\mathrm{C}-\mathrm{C}}=1.42 \AA$ is the carbon-carbon distance ${ }^{8}$. We choose a chemical potential $\mu=0.5 \mathrm{eV}$, which sets the Dirac point energy $E_{\mathrm{D}}=-0.5 \mathrm{eV}$ relative to $\mu$. This choice is motivated by the fact that typical graphene samples on substrates are doped, and that states both below and above the Dirac point energy are occupied in the initial equilibrium states and therefore nicely visible in the tr-ARPES spectra.

The simulation parameters for the pump-probe setup are as follows: The pump laser field has frequency $\Omega=1.5 \mathrm{eV}$, unless denoted otherwise, implying oscillation periods of $2.58 \mathrm{fs}$. Its temporal width is $\sigma_{\text {pump }}=165 \mathrm{fs}$. We vary the peak vector potential $A_{\max }=0.10 \ldots 1.00$ in units of $\mathrm{a}_{\mathrm{C}-\mathrm{C}}$. This corresponds to peak electric field strengths $E_{\max }=\Omega A_{\max }$ of $106 \ldots 1,060 \mathrm{mV} \AA^{-1}$ for $\Omega=1.5 \mathrm{eV}$ and the graphene lattice parameters. The photoemission probe-pulse has a width $\sigma_{\text {probe }}=26 \mathrm{fs}$. This choice of parameters is motivated by the hierarchy of time scales in the system: The oscillation period for the pump laser light, the temporal width of the probe-pulse, which controls the time and energy resolution for the tr-ARPES signal, and the temporal width of the pump-pulse, which controls the nonequilibrium state and ensures a well-defined center frequency for the pump-pulse.

Model and time evolution. Our goal is to obtain the lesser Green function matrix $\mathcal{G}^{<}\left(\mathbf{k}, t, t^{\prime}\right)$ in $2 \times 2$ orbital space (see below) with matrix elements

$$
\mathcal{G}_{\alpha \beta}^{<}\left(\mathbf{k}, t, t^{\prime}\right) \equiv i\left\langle\alpha_{\mathbf{k}}^{\dagger}(t) \beta_{\mathbf{k}}\left(t^{\prime}\right)\right\rangle
$$

where $\alpha_{\mathbf{k}}^{\dagger}\left(\beta_{\mathbf{k}}\right)$ is a creation (annihilation) operator for a fermion at momentum $\mathbf{k}$ in orbital $\alpha(\beta) \in\{a, b\}$. As shown below, the photocurrent and pseudospin contents are computed from these lesser Green functions.

Including the field via Peierls substitution, the time-dependent Hamiltonian for $\mathcal{A}$ and $\mathcal{B}$ sublattices with corresponding orbitals $a$ and $b$ reads

$$
\mathcal{H}(t)=\sum_{\mathbf{k}}\left(\begin{array}{ll}
a_{\mathbf{k}}^{\dagger} & b_{\mathbf{k}}^{\dagger}
\end{array}\right)\left(\begin{array}{cc}
0 & g(\mathbf{k}-\mathbf{A}(t)) \\
g^{*}(\mathbf{k}-\mathbf{A}(t)) & 0
\end{array}\right)\left(\begin{array}{l}
a_{\mathbf{k}} \\
b_{\mathbf{k}}
\end{array}\right),
$$

with the Hamiltonian matrix elements

$$
\begin{aligned}
g(\mathbf{k})= & V\left[2 \cos \left(\frac{\sqrt{3} k_{x}}{2}\right) \cos \left(\frac{k_{y}}{2}\right)+\cos \left(k_{y}\right)\right. \\
& \left.+i\left(-2 \cos \left(\frac{\sqrt{3} k_{x}}{2}\right) \sin \left(\frac{k_{y}}{2}\right)+\sin \left(k_{y}\right)\right)\right],
\end{aligned}
$$

where $V=2.8 \mathrm{eV}$ is the nearest-neighbour hopping matrix element matching the graphene bandwidth and Dirac point velocity. In equilibrium, the Hamiltonian has two Dirac points at momenta $K$ and $K^{\prime}$ given by $( \pm 4 \pi /(3 \sqrt{3}), 0) \approx( \pm 2.4184,0)$, where momenta and the vector field $\mathbf{A}(t)$ are measured in multiples of the inverse of the carbon-carbon distance $\mathrm{a}_{\mathrm{C}-\mathrm{C}} 8$.

It is convenient to define the Hamiltonian matrix for momentum $\mathbf{k}$ in orbital basis,

$$
\mathcal{H}(\mathbf{k}, t)=\left(\begin{array}{cc}
0 & g(\mathbf{k}-\mathbf{A}(t)) \\
g^{*}(\mathbf{k}-\mathbf{A}(t)) & 0
\end{array}\right) .
$$

In the absence of a driving field, this Hamiltonian is diagonalized by a rotation $\mathcal{R}(\mathbf{k})$ at $t=0$, which is a time before the pump-pulse is turned on. Note that $t=0$ is used here as a notation for the earliest real time we consider, not to be confused with zero delay time $\Delta t \equiv t_{\mathrm{pr}}-t_{\mathrm{p}}=0$, which refers to the time where the Gaussian pump-pulse envelope is maximal. For later times, the given rotation does not diagonalize the Hamiltonian except for accidental cases where the gauge field is an integer multiple of a reciprocal lattice vector.

The computation of double-time propagators requires the evaluation of the time evolution operators

$$
\mathcal{U}\left(\mathbf{k}, t, t^{\prime}\right)=\mathcal{T} \exp \left(-i \int_{t^{\prime}}^{t} \mathcal{H}(\mathbf{k}, \bar{t}) \mathrm{d} \bar{t}\right)
$$

Since $\mathcal{U}(\mathbf{k}, t)$ at different times do not commute with each other, the time ordering $\mathcal{T}$ in $\mathcal{U}\left(\mathbf{k}, t, t^{\prime}\right)$ is taken into account by discretization of the real-time axis and multiplication of the resulting time-step evolution operators. We then obtain the time evolution operator as $2 \times 2$ matrices in band basis,

$$
\mathcal{U}\left(\mathbf{k}, t, t^{\prime}\right) \approx \prod_{j=1}^{N_{t, t^{\prime}}} \exp [-i \mathcal{H}(\mathbf{k}, t-j \delta t / 2) \delta t]
$$


where $N_{t, t^{\prime}}$ is the number of fine time steps of size $\delta t$ between $t$ and $t^{\prime}$, and the product is understood as time ordered with later times to the left.

The lesser Green function matrix results from

$$
\begin{gathered}
\mathcal{G}^{<}\left(\mathbf{k}, t, t^{\prime}\right)=\mathcal{U}(\mathbf{k}, t, 0)^{\dagger} \mathcal{G}^{<}(\mathbf{k}, 0,0) \mathcal{U}\left(\mathbf{k}, t^{\prime}, 0\right), \\
\mathcal{G}^{<}(\mathbf{k}, 0,0)=\mathcal{R}(\mathbf{k}) i \mathcal{N}(\mathbf{k}) \mathcal{R}(\mathbf{k})^{\dagger},
\end{gathered}
$$

where time 0 refers to an initial time where the system is in equilibrium before the pump-pulse is turned on, and $\mathcal{N}(\mathbf{k})$ is the time-independent diagonal matrix of initial equilibrium band occupation with diagonal elements $f\left(\epsilon_{1}(\mathbf{k})\right)$ and $f\left(\epsilon_{2}(\mathbf{k})\right)$ corresponding to Fermi function filling for the two energy eigenvalues.

Floquet spectra. The Floquet spectra shown in Figs 2 and 3 are calculated from the Floquet Hamiltonian corresponding to equation (3):

$$
H_{F}=-\sum_{m \alpha} m \Omega|m, \alpha\rangle\langle m, \alpha|+\sum_{m n}\left[g_{m-n}(\mathbf{k})|m, \mathcal{A}\rangle\langle n, \mathcal{B}|+\text { h.c. }\right]
$$

where $g_{m-n}(\mathbf{k})$ are the Fourier series expansion coefficients of $g(\mathbf{k}-\mathbf{A}(t))$ :

$$
\begin{gathered}
g_{m-n}(\mathbf{k})=\frac{\Omega}{2 \pi} \int_{0}^{\frac{2 \pi}{2}} d t e^{i(m-n) \Omega t} g(\mathbf{k}-\mathbf{A}(t)) \\
=\left[e^{i\left[k_{y}+\frac{7 \pi}{2}(m-n)\right]}+e^{i\left[\frac{\sqrt{3}}{2} k_{x}-\frac{1}{2} k_{y}+\frac{\pi}{6}(m-n)\right]}+e^{-i\left[\frac{\sqrt{3}}{2} k_{x}+\frac{1}{2} k_{y}-\frac{5 \pi}{6}(m-n)\right]}\right] J_{m-n}(A)
\end{gathered}
$$

Here $J_{n}(A)$ is the Bessel function of the first kind. As the pump frequencies considered in this work are small with respect to the electronic bandwidth, the corresponding spectrum must be evaluated numerically via truncation of the full Floquet Hamiltonian. In practice, we achieve convergence for $|m| \leq 40$.

Time-resolved ARPES formalism. The computation of the time-resolved photocurrent involves normalized Gaussian probe-pulse shape functions $s_{\sigma \text { probe }}(t)$ of width $\sigma_{\text {probe }}$ centred around time $t$. In the Hamiltonian gauge, the photocurrent (tr-ARPES intensity) at momentum $\mathbf{k}$, binding energy $\omega$ and pump-probe delay time $\Delta t \equiv t_{\mathrm{pr}}-t_{\mathrm{p}}$ is then obtained from ${ }^{27}$

$$
I(\mathbf{k}, \omega, \Delta t)=\operatorname{Im} \sum_{a} \int \mathrm{d} t_{1} \int \mathrm{d} t_{2} s_{\sigma_{\text {probe }}}\left(t_{\mathrm{pr}}-t_{1}\right) s_{\sigma_{\mathrm{probe}}}\left(t_{\mathrm{pr}}-t_{2}\right) e^{i \omega\left(t_{1}-t_{2}\right)} G_{a a}^{<}\left(\mathbf{k}, t_{1}, t_{2}\right) .
$$

In the main text (Fig. 2) we show tr-ARPES spectra at peak field strength with false colour plots of the tr-ARPES intensity $I(\mathbf{k}, \omega, \Delta t=0)$, that is, intensity variations as a function of binding energy $\omega$ along selected momentum cuts $\mathbf{k}$. The location of energy bands $E(\mathbf{k})$ can be obtained from the maxima in the ARPES intensity as a function of binding energy at constant momentum, the so-called energy distribution curves. As seen in Fig. 2 of the main text, these bands are in excellent agreement with quasi-static Floquet bands, whose calculation is described below.

The photocurrent as defined in equation (12) is computed from the lesser Green function in a fixed gauge. We would like to point out that this quantity is not gauge-invariant. In fact, the general definition of a gauge-invariant photocurrent that fulfills the positivity criterion $I(\mathbf{k}, \omega, \Delta t) \geq 0$ for all $\mathbf{k}, \omega, \Delta t$ in the presence of a field is an outstanding research problem ${ }^{43}$. The problem likely lies in the neglect of photoemission matrix elements, which can be momentum and field dependent. The photocurrent according to equation (12) manifestly fulfills the positivity criterion. In addition, it also matches the Floquet band structure, as shown in the present work. The Floquet band structure is not gauge-invariant either. Importantly, general conclusions drawn from the analysis of Floquet sidebands, level crossings and gap closings are valid even in a fixed-gauge calculation. This is due to the fact that the time-resolved, momentum-integrated photoemission spectrum (tr-PES) is always manifestly gauge-invariant and positive. The tr-PES signal is obtained by integrating the tr-ARPES spectrum in any gauge over all momenta. Hence, conclusions about the presence or absence of energy gaps can be drawn even in a gauge-variant formalism.

\section{References}

1. Fausti, D. et al. Light-induced superconductivity in a stripe-ordered cuprate. Science 331, 189-191 (2011).

2. Sobota, J. A. et al. Ultrafast optical excitation of a persistent surface-state population in the topological insulator $\mathrm{Bi}_{2} \mathrm{Se}_{3}$. Phys. Rev. Lett. 108, 117403 (2012).

3. Caviglia, A. D. et al. Ultrafast strain engineering in complex oxide heterostructures. Phys. Rev. Lett. 108, 136801 (2012).

4. Konig, M. et al. Quantum spin Hall insulator state in HgTe quantum wells. Science 318, 766-770 (2007).

5. Wang, Y. H., Steinberg, H., Jarillo-Herrero, P. \& Gedik, N. Observation of Floquet-Bloch states on the surface of a topological insulator. Science 342, 453-457 (2013).

6. Hasan, M. Z. \& Kane, C. L. Colloquium: Topological insulators. Rev. Mod. Phys. 82, 3045-3067 (2010).
7. Bernevig, B. A., Hughes, T. L. \& Zhang, S.-C. Quantum spin Hall effect and topological phase transition in HgTe quantum wells. Science 314, 1757-1761 (2006).

8. Castro Neto, A. H., Guinea, F., Peres, N. M. R., Novoselov, K. S. \& Geim, A. K. The electronic properties of graphene. Rev. Mod. Phys. 81, 109-162 (2009).

9. Haldane, F. D. M. Model for a Quantum Hall Effect without Landau Levels: Condensed-Matter Realization of the "Parity Anomaly". Phys. Rev. Lett. 61, 2015-2018 (1988).

10. Oka, T. \& Aoki, H. Photovoltaic Hall effect in graphene. Phys. Rev. B 79, 081406 (2009).

11. Kitagawa, T., Berg, E., Rudner, M. \& Demler, E. Topological characterization of periodically driven quantum systems. Phys. Rev. B 82, 235114 (2010).

12. Kitagawa, T., Oka, T., Brataas, A., Fu, L. \& Demler, E. Transport properties of nonequilibrium systems under the application of light: Photoinduced quantum Hall insulators without Landau levels. Phys. Rev. B 84, 235108 (2011).

13. Lindner, N. H., Refael, G. \& Galitski, V. Floquet topological insulator in semiconductor quantum wells. Nat. Phys. 7, 490-495 (2011).

14. Gu, Z., Fertig, H. A., Arovas, D. P. \& Auerbach, A. Floquet spectrum and transport through an irradiated graphene ribbon. Phys. Rev. Lett. 107, 216601 (2011).

15. Calvo, H. L., Pastawski, H. M., Roche, S. \& Foa Torres, L. E. F. Tuning laser-induced band gaps in graphene. Appl. Phys. Lett. 98, 232103 (2011)

16. Dora, B., Cayssol, J., Simon, F. \& Moessner, R. Optically engineering the topological properties of a spin Hall insulator. Phys. Rev. Lett. 108, 056602 (2012).

17. Suarez Morell, E. \& Foa Torres, L. E. F. Radiation effects on the electronic properties of bilayer graphene. Phys. Rev. B 86, 125449 (2012).

18. Rudner, M. S., Lindner, N. H., Berg, E. \& Levin, M. Anomalous edge states and the bulk-edge correspondence for periodically driven two-dimensional systems. Phys. Rev. X 3, 031005 (2013).

19. Iadecola, T. et al. Materials design from nonequilibrium steady states: driven graphene as a tunable semiconductor with topological properties. Phys. Rev. Lett. 110, 176603 (2013).

20. Perez-Piskunow, P. M., Usaj, G., Balseiro, C. A. \& Foa Torres, L. E. F. Floquet chiral edge states in graphene. Phys. Rev. B 89, 121401 (2014).

21. Grushin, A. G., Gomez-Leon, A. \& Neupert, T. Floquet fractional Chern insulators. Phys. Rev. Lett. 112, 156801 (2014).

22. Fregoso, B. M., Wang, Y. H., Gedik, N. \& Galitski, V. Driven electronic states at the surface of a topological insulator. Phys. Rev. B 88, 155129 (2013).

23. Kundu, A., Fertig, H. A. \& Seradjeh, B. Effective theory of Floquet topological transitions. Phys. Rev. Lett. 113, 236803 (2014).

24. Dehghani, H., Oka, T. \& Mitra, A. Dissipative Floquet topological systems. Phys. Rev. B 90, 195429 (2014).

25. Dahlhaus, J. P., Fregoso, B. M. \& Moore, J. E. Magnetization signatures of lightinduced quantum Hall edge states. Preprint at http://arxiv.org/abs/1408.6811 (2014).

26. Thouless, D. J., Kohmoto, M., Nightingale, M. P. \& den Nijs, M. Quantized Hall conductance in a two-dimensional periodic potential. Phys. Rev. Lett. 49, 405-408 (1982).

27. Freericks, J. K., Krishnamurthy, H. R. \& Pruschke, T. Theoretical description of time-resolved photoemission spectroscopy: application to pump-probe experiments. Phys. Rev. Lett. 102, 136401 (2009).

28. Sentef, M. et al. Examining electron-boson coupling using time-resolved spectroscopy. Phys. Rev. X 3, 041033 (2013).

29. Min, H. \& MacDonald, A. H. Chiral decomposition in the electronic structure of graphene multilayers. Phys. Rev. B 77, 155416 (2008).

30. Kumar, A. \& Nandkishore, R. Flat bands with Berry curvature in multilayer graphene. Phys. Rev. B 87, 241108 (2013).

31. Zhang, H., Liu, C.-X. \& Zhang, S.-C. Spin-orbital texture in topological insulators. Phys. Rev. Lett. 111, 066801 (2013).

32. Cayssol, J., Dora, B., Simon, F. \& Moessner, R. Floquet topological insulators. Phys. Status Solidi RRL 7, 101-108 (2013).

33. Johannsen, J. C. et al. Direct view of hot carrier dynamics in graphene. Phys. Rev. Lett. 111, 027403 (2013).

34. Gierz, I. et al. Snapshots of non-equilibrium Dirac carrier distributions in graphene. Nat. Mater. 12, 1119-1124 (2013).

35. Xiao, D., Liu, G.-B., Feng, W., Xu, X. \& Yao, W. Coupled spin and valley physics in monolayers of $\mathrm{MoS}_{2}$ and other group-VI dichalcogenides. Phys. Rev Lett. 108, 196802 (2012).

36. Wu, S. et al. Electrical tuning of valley magnetic moment through symmetry control in bilayer $\mathrm{MoS}_{2}$. Nat. Phys. 9, 149-153 (2013).

37. Xu, X., Yao, W., Xiao, D. \& Heinz, T. F. Spin and pseudospins in layered transition metal dichalcogenides. Nat. Phys. 10, 343-350 (2014).

38. Tahir, M., Manchon, A. \& Schwingenschlogl, U. Photoinduced quantum spin and valley hall effects, and orbital magnetization in monolayer $\mathrm{MoS}_{2}$. Phys. Rev. B 90, 125438 (2014).

39. Uehlinger, T. et al. Double transfer through Dirac points in a tunable honeycomb optical lattice. Eur. Phys. J. Special Topics 217, 121-133 (2013). 
40. Tarruell, L., Greif, D., Uehlinger, T., Jotzu, G. \& Esslinger, T. Creating, moving and merging Dirac points with a Fermi gas in a tunable honeycomb lattice. Nature 483, 302-305 (2012).

41. Jotzu, G. et al. Experimental realization of the topological Haldane model with ultracold fermions. Nature 515, 237-240 (2014).

42. Wallace, P. R. The band theory of graphite. Phys. Rev. 71, 622-634 (1947).

43. Freericks, J. K., Krishnamurthy, H. R., Sentef, M. A. \& Devereaux, T. P. Gauge invariance in the theoretical description of time-resolved angle-resolved pump/probe photoemission spectroscopy. Preprint at http://arxiv.org/abs/ 1403.7585 (2014).

\section{Acknowledgements}

We acknowledge helpful discussions with Patrick Kirchmann, Sri Raghu, Xiao-Liang Qi, Shou-Cheng Zhang, and Bruce Normand. This work was supported by the Department of Energy, Office of Basic Energy Sciences, Division of Materials Sciences and Engineering under Contract Nos. DE-AC02-76SF00515 (Stanford/SIMES), DE-FG0208ER46542 (Georgetown) and DE-SC0007091 (for the collaboration). Computational resources were provided by the National Energy Research Scientific Computing Center supported by the Department of Energy, Office of Science, under Contract No. DE- AC02-05CH11231. J.K.F. was also supported by the McDevitt bequest at Georgetown. A.F.K. was supported by the Laboratory Directed Research and
Development Program of Lawrence Berkeley National Laboratory under U.S. Department of Energy Contract No. DE-AC02-05CH11231.

\section{Author contributions}

M.A.S. developed the computer code and performed the ARPES and pseudospin calculations. A.F.K. assisted in developing the computer code for the ARPES calculations. M.C. developed the computer code and performed the Floquet calculations, and T.O. assisted in developing the computer code for the Floquet calculations. M.A.S., M.C., A.F.K., B.M., J.K.F. and T.P.D. wrote the manuscript. T.P.D. and J.K.F. are responsible for project planning.

\section{Additional information}

Supplementary Information accompanies this paper at http://www.nature.com/ naturecommunications

Competing financial interests: The authors declare no competing financial interests.

Reprints and permission information is available online at http://npg.nature.com/ reprintsandpermissions/

How to cite this article: Sentef, M.A. et al. Theory of Floquet band formation and local pseudospin textures in pump-probe photoemission of graphene. Nat. Commun. 6:7047 doi: 10.1038/ncomms8047 (2015). 